\title{
The impact of integrated obstetric and neonatal services on utilization of postpartum maternal health care services
}

\author{
Fisun Vural, ${ }^{1}$ Filiz Yildirim, ${ }^{2}$ Birol Vural ${ }^{3}$ \\ ${ }^{1}$ Department of Obstetrics and Gynecology, Haydarpasa Numune Training and Research Hospital, Istanbul, Turkey \\ ${ }^{2}$ Department of Health Care Management, Golcuk Necati Celik State Hospital, Kocaeli, Turkey \\ ${ }^{3}$ Department of Obstetrics and Gynecology, Kocaeli University Faculty of Medicine, Kocaeli, Turkey
}

\begin{abstract}
OBJECTIVE: Postnatal care is an important issue in maintaining and promoting maternal and neonatal health. However, utilization of postpartum maternal health care services is at a low rate in many countries. This study was aimed to investigate the impact of integrated obstetric and neonatal services on utilization rates of postnatal health care service among mothers.
\end{abstract}

METHODS: This study was performed among a total of 4193 mothers who gave birth at Maternity Unit of Golcuk Necati Celik State Hospital of Kocaeli Province between 2010 and 2013. All mothers were called back to postnatal care clinic (PNC) for newborn hearing test (NHT) screenings, neonatal and maternal care within two weeks after delivery. The deliveries after, $(n=3093)$ and before $(n=1100)$ utilization of integrated services were compared as for postnatal service utilization rates.

RESULTS: Utilization rates of neonatal health care, NHT and postpartum maternal health care services significantly increased after implementation of integrated services $(p<0.0001)$. Especially maternal service utilization rates increased from $34 \%$ to 99 percent.

CONCLUSION: Integration of newborn and maternal health care services as a unit increases the utilization of PNC services.

Keywords: Health care; maternal; neonatal; perinatal; postnatal care; postpartum.

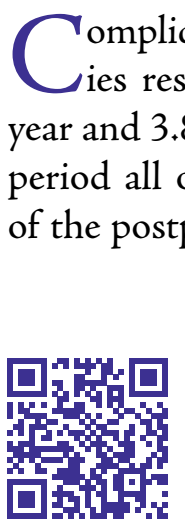

and morbidity rates related to maternal and child care [1]. Perinatal infections, asphyxia, birth complications, and premature births are the commonest causes of neonatal death. Postpartum bleedings, infections, and high blood pressure are the most

Received: March 01, 2015 Accepted: August 25, 2015 Online: September 25, 2015

Correspondence: Dr. Fisun VURAL. Haydarpasa Numune Egitim ve Arastirma Hastanesi, Kadin Hastaliklari ve Dogum Klinigi, Istanbul, Turkey.

Tel: +90 532 - 4173292 e-mail: fisunvural@yahoo.com.tr

(c) Copyright 2015 by Istanbul Northern Anatolian Association of Public Hospitals - Available online at www.kuzeyklinikleri.com 
common problems associated with maternal death during the first month of life $[4,5,6]$.

Postnatal period is a critical transition and adaptation time for a woman and her newborn. Postnatal care (PNC) is an important issue in maintaining and promoting maternal and neonatal health. It provides an opportunity to discuss neonatal care, promote exclusive breastfeeding, offer counseling for family planning, immunizations, and recognize early maternal and newborn problems $[1,2]$. However, underutilization of health care services is a common problem. Underutilization may be secondary to the inability to access health care systems or inequality in the distribution of health care services, and the fact that mothers often fail to give priority to health care after labor $[3,4]$. Therefore, development of strategies, and increasing the utilization of obstetric care services are essential $[1,7]$.

Turkey has a population of 76.667 .864 with a relatively young age structure. The number of annual births is 1.262698 . Neonatal and maternal mortality rates in Turkey have declined substantially over the past ten years $[8,9,10,11]$. According to Demographic Health Survey (DHS) data (2008), maternal deaths dropped from 28.5 to $19.5 / 100.000$ and a neonatal mortality fell from 26 to $10 / 1000$ [10]. Ninety-seven percent of women received antenatal care from a medical provider which was delivered in a health care facility. These women received postnatal checkups (94\%), and postnatal care (PNC) within 24 hours $(80.2 \%)$ or 40 days after delivery $(13.1 \%)$, while $6.5 \%$ of the women had not benefited from PNC. Ninety-five percent of infants received PNC from medical professionals [12]. Every year $54 \%$ of all pediatric mortalities occur within the neonatal period, while $87 \%$ of them within the first week of their lives $[7,9,10,11]$. Approximately half of all maternal deaths are avoidable $[9,10]$. These figures show the importance of PNC for early recognition and prevention of maternal and neonatal problems. Moreover, PNC includes support of mothers for exclusive breastfeeding and contraception.

In Turkey, although a significant success has been achieved within the last decade by decreasing maternal and neonatal mortality rates by increas- ing institutional deliveries and national immunization programs [12], maternal PNC service utilization rates after delivery still needs to be improved. To the best of our knowledge, maternal postnatal care after hospital discharge and service utilization are not well investigated in Turkey. This is the first preliminary report of integrated health services in Turkey. When this hospital-based quality study planned, maternal and neonatal postnatal services were integrated and accepted as a unit. This paper was aimed to investigate whether the integration of neonatal and maternal care services and NHT screening programs increase PNC service utilization rates of mothers.

\section{MATERIALS AND METHODS}

\section{Study area}

The province of Kocaeli is one of the densely populated cities and it is located in the northwestern part of Turkey. Golcuk is a district in this province. The Golcuk Necati Celik State Hospital has the only maternity unit in this region and every year an average of 1300 deliveries are performed in this babyfriendly institution.

\section{Study design}

This prospective study was performed at Golcuk Necati Celik State Hospital, Kocaeli, Turkey between March 1, 2010 and September 31, 2013. A total of 4193 live births were included in the study. Postnatal care clinic of this hospital was launched on July 2011. This service accepts maternal and neonatal care as a unit. Postnatal care of mothers and infants and NHT screenings were performed in this clinic. Before integration of the neonatal, and obstetric clinics (between March 1, 2010 and June $30,2011) 1100$, while after integration (between July 1, 2011 and September 31, 2013) 3093 women had given birth. The deliveries to be investigated before the integration were selected from all births of the preceding year excluding patients with unavailable data and still births.

This quality report examined the effect of integration of maternal and neonatal care on utiliza- 


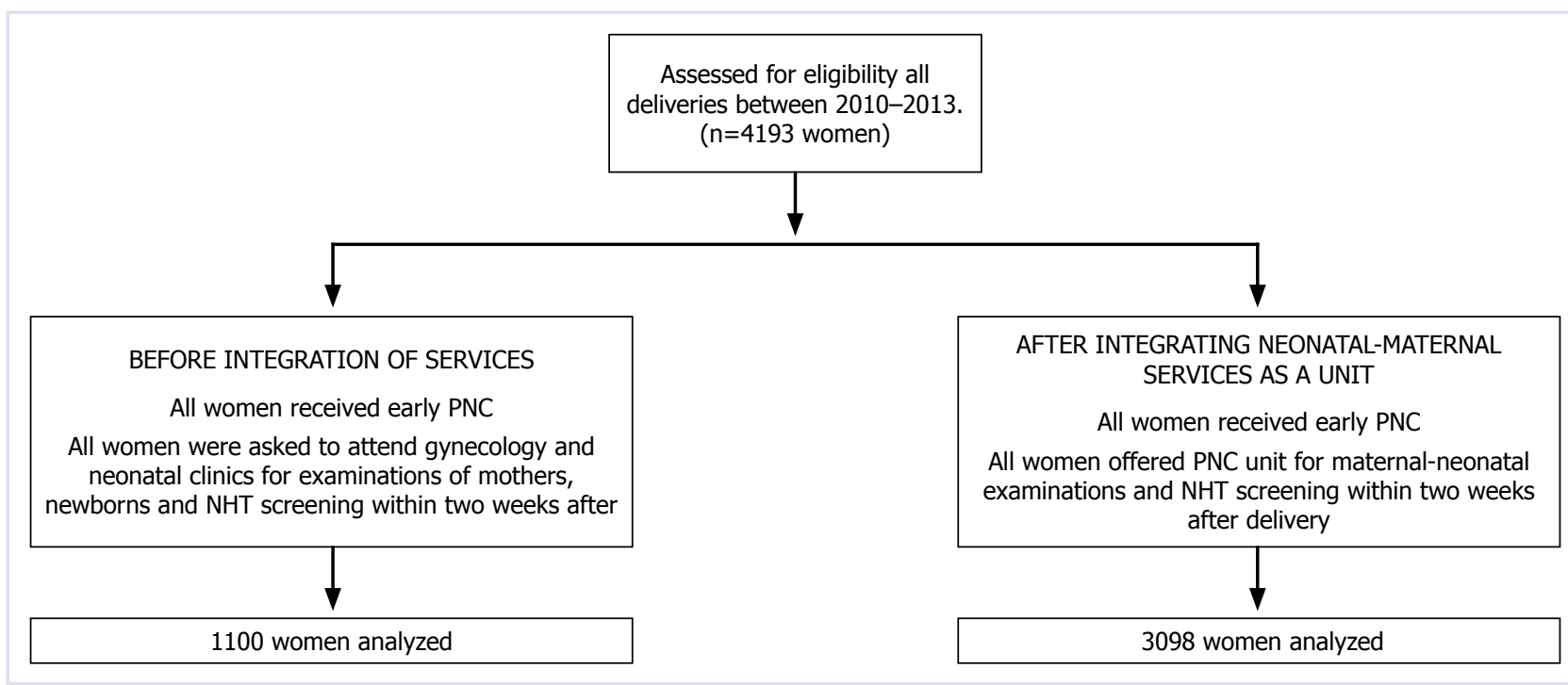

FIGURE 1. Recruitment protocol flow diagram.

tion of health services. The primary outcome measure was the attendance rate of mothers to hospital health care services after delivery. This integrated health service project was audited by Kocaeli Provincial Health Directorate of Turkish Ministry of Health. This study was approved by the Ethic Committee of Kocaeli University, Faculty of Medicine, Kocaeli Turkey.

\section{Postnatal care}

Early PNC: All postpartum women (before or after establishment of integrating services) received PNC within 24 hours of delivery, and gynecological examinations performed before hospital discharge. Midwives informed the women about the importance of PNC, family planning, NC, and breastfeeding. Before integrating services, all women were asked to return to gynecology and newborn clinics for PNC service visits within two weeks after delivery both for maternal and neonatal physical examination and NHT screening. After integrating services all women were requested to return to $\mathrm{PNC}$ unit within two weeks after delivery.

PNC at two weeks after delivery: Firstly mothers attended PNC clinic and they were evaluated by midwives (NS). Then blood pressure measurements, complete blood cell counts, and urinalysis were performed routinely. Presence of vaginal bleed- ing (retained placenta, endometritis, lacerations, hematoma, and coagulation problems), urinary problems, and fecal incontinence were questioned. Screenings were performed for iron supplementation, nutritional status, counseling, and depression. Mothers received information about neonatal and postnatal care, breastfeeding, and nutrition. Patients were consulted to a gynecologist if there was any gynecological problem or psychiatrist if they had higher Edinburg Depression scores. Then, a routine pediatric examination was performed along with NHT screening. An otoacoustic emission test was given to all newborns. High-risk babies underwent brainstem evoked response audiometry (BERA) tests. The PNC visit protocol was the same before and after integration of services, but the mother and her child attended to the PNC unit after integration of services rather than attending gynecology and neonatal clinics separately. Figure 1 shows the flow diagram of the study protocol.

\section{Data analysis}

Statistical Package for Social Sciences (SPPS) 16 software (SPSS Inc., IL. Chicago, USA) was used for statistical analysis. All data were bidirectionally evaluated within $95 \%$ confidence interval. A p value of $<0.05$ was accepted as the statistically significant level. Data were expressed as means, standard devi- 
TABLE 1. Characteristic findings of the participants

\begin{tabular}{lccc} 
& Minimum & Maximum & Mean \pm SD \\
\hline Maternal age & 8 & 49 & $28.2 \pm 5.4$ \\
Previous pregnancies (n) & 1 & 10 & $2.4 \pm 1.2$ \\
Previous births (n) & 1 & 10 & $1.8 \pm 1.2$ \\
Unintended pregnancies (D\&C) (n) & 0 & 4 & $0.19 \pm 0.5$ \\
Spontaneous abortions (n) & 0 & 5 & $0.02 \pm 0.3$ \\
Antenatal care visits (n) & 0 & 9 & $4.86 \pm 0.5$ \\
Postnatal care visits (n) & 1 & 3 & $1.5 \pm 0.5$ \\
First antenatal visit (month) & 2 & 9 & $5.9 \pm 0.8$ \\
\hline
\end{tabular}

SD: Standard deviation; D\&C: Dilatation and curettage.

ations, and percentages. The $\chi^{2}$-test was used for the comparison of different variables. The relationships between the data were evaluated using Spearman's correlation. Logistic regression analysis was used for correlates of relevant factors.

\section{RESULTS}

A total of 4193 women aged between $18-49$ years were included in the study. The mean age \pm SD of the women was $28.2 \pm 5.4$ years. The great majority (91.3\%; $n=3830)$ of the mothers had visited their obstetricians at least once during the prenatal period. Vaginal deliveries, and cesarean sections were performed on $64.4 \%(n=2700)$, and $35.6 \%$ of the mothers, respectively. Table 1 presents the characteristic findings of the participants before and af- ter integration. Sociodemographic findings before, and after integration of services were nearly similar $(\mathrm{p}>0.05)$.

Before the integration: A total of 1100 live births with available data were included in the study. The attendance rate for NHT screenings was $92 \%(n=1012)$, while $90 \%(n=990)$ of them had undergone neonatal examinations by a pediatrician. A total of 374 women were admitted to PNC service within two weeks after delivery (34\%). Table 2 displays the attendance rates of women and newborns to postnatal care and neonatal care services.

After the integration: A total of 3093 live births were included in the study. The attendance rate for NHT screenings, pediatric examination and maternal care was $99.03 \%(n=3063)$. The outcomes of the study are compared in Table 2. The utilization

TABLE2. Utilization rates of PNC services before and after integration of services

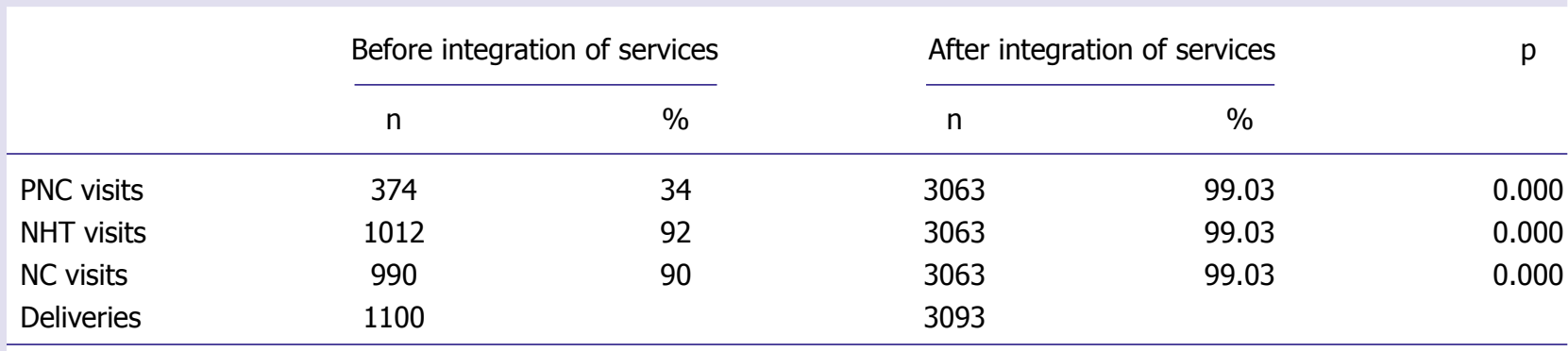

PNC: Postnatal care; NHT: Newborn hearing test; NC: Neonatal care. 


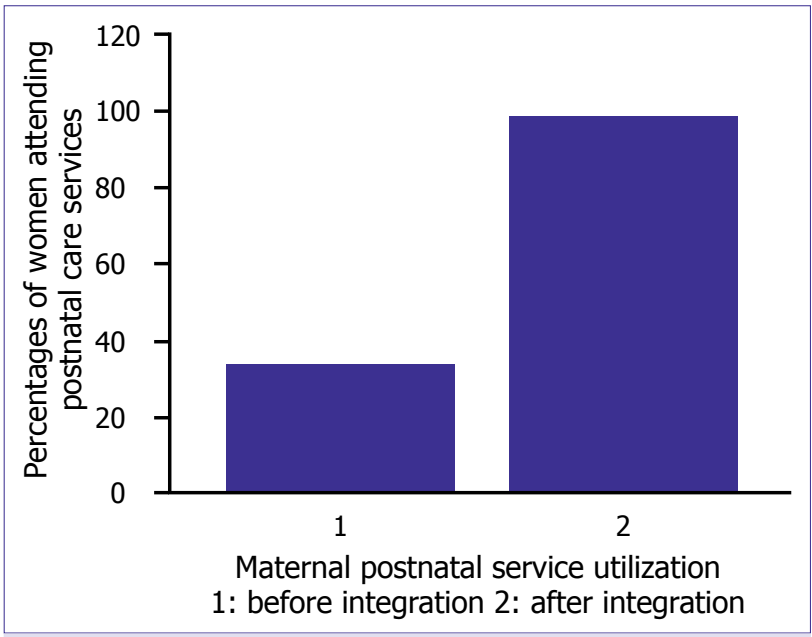

FIGURE 2. Maternal PNC healthcare service utilization rates before and after integration of services $(p<0.05)$.

rate of postnatal care clinics significantly increased after integration of services $(\mathrm{p}<0.05)$.

Figure 2 shows the comparative data of postnatal service utilization before and after the integration $(\mathrm{p}<0.05)$.

Factors Affecting PNC utilization before integration: Maternal age $(r=0.321, p=0.001)$, number of antenatal $(r=0.281, p=0.004)$ and neonatal care visits $(r=0.681, p=0.00)$ were the factors significantly related to PNC visits attended by mothers. The correlated factors were accepted as independent variables and a logistic regression analysis was conducted to predict factors affecting PNC utilization. Table 3 shows the results of the logistic regression analysis. According to this equation, neonatal care $(\operatorname{Exp}(B): 1.72, p=0.001, C I \div 1.2-2.4)$ was the only independent factor affecting maternal health services uptake.

\section{DISCUSSION}

Primary health care interventions can prevent many complications during the postpartum period. Timely, efficient, and skilled healthcare support can allow for early detection and management of problems [8]. Moreover, it provides support for exclusive breastfeeding and solutions for maternal social, physical and mental problems. However, underutilization of health services by mothers after hospital discharge is a common problem. This paper examined the impact of the integrated neonatal and maternal services on maternal PNC service utilization. The results of this study showed that integrated services significantly increased maternal postnatal service use.

The DHS 2008 results of Turkey showed that the majority of the maternal and neonatal mortality cases is observed in the first week of the postpartum period [9]. Fifty-two percent of maternal deaths are avoidable, and most of them occur in the third trimester or within the 48 hours of delivery $[7,9]$. The perinatal death rate is $19 / 1000$ births, and the neonatal mortality rate is $13 / 1000$ in Turkey [10]. Neonatal and maternal mortality rates in Turkey have declined over the past ten years [10]. Prematurity and its complications are the most common causes of neonatal deaths in Turkey, followed by congenital anomalies, infections, and asphyxia [7,9].

The majority of these neonatal problems has been observed in the early neonatal period [9]. The primary intervention to decrease maternal and neonatal mortality is increasing the number of deliveries performed in the health care facilities. All people under 18 years of age, and pregnancies are registered in the General Health Insurance system in

TABLE 3. Logistic regression analysis of associated factors of maternal postpartum health care uptake

\begin{tabular}{lccc} 
Factors & Relative risk & Significance & Confidence interval (95\%) \\
\hline Neonatal care visits & 1.72 & 0.001 & $1.2-2.4$ \\
Antenatal visits & 1.33 & 0.333 & $0.743-2.4$ \\
Maternal age & 0.74 & 0.341 & $0.4-1.3$
\end{tabular}


Turkey. The institutional deliveries have increased and approximately $97 \%$ of women delivered their babies in health care facilities. The duration of hospitalization is 24 hours for vaginal, and 48 hours for cesarean section deliveries in compliance with Declaration of Safer Motherhood by Turkish Ministry of Health $[7,9,10,11,12]$.

The disparity in the use of maternity care services has been examined in various settings. Prior investigations have demonstrated that education of women is one of the most important determinants of maternity service use [13, 14, 15, 16, 17, 18, 19]. According to Demographic Health Survey (2013) reports of Turkey, only $5 \%$ of the mothers are receiving obstetric care during two weeks postpartum after hospital discharge. When our results (prior to integration) are compared with those of DHS 2013 reports of Turkey, increases in PNC utilization rates $(34 \%)$ can be seen for all mothers who were counselled during immediate postpartum period. These results suggested us that postnatal education of mothers immediately after birth is an essential primary step to increase rates of maternity service use. These findings confirmed the prior investigations which suggested that education of women is one of the most important determinants of PNC utilization $[13,14,15,16,17,18,19]$. However, rates of maternal service utilization were still low, in consideration of higher rates of attendance at pediatric clinics and NHT screening tests. This result suggested us that additional effort is needed to increase the rates of postnatal service utilization. The discrepancy in maternal and neonatal PNC rates suggested us that the majority of women had given priority to take care of their infants, but they did not attend maternal visits concerning postnatal care. For this reason, the factors related to postnatal service utilization before integration were analyzed by logistic regression tests. Neonatal care visits were the only independent factors affecting maternal service utilization rates $(p<0.05)$. Congenital hearing loss is a serious public health concern that affects the speaking, language and learning abilities of the babies [20]. The National Newborn Hearing Screening Program (NNHSP) has been conducting relevant studies in Turkey since 2008 [21]. In this study, we integrated neonatal care with NHT screening and maternal care. The results of this report have demonstrated that the integration of neonatal and maternal services as a unit after delivery have given an opportunity to assess maternal outcomes.

The factors affecting PNC utilization rates have been studied in various countries $[22,23,24,25,26$, $27,28]$. The knowledge of women about the importance of postnatal care and inequities in health care systems are the main factors related to utilization of these services. The study from Nepal showed that the majority of the women did not seek postnatal care. They found that antenatal care, institutional deliveries, awareness and access to services were the determinants of PNC [27]. However, the majority of the studies was performed in underdeveloped countries struggling with inequietes in the allocation of the health care services and increased home deliveries [25, 26, 27, 28]. Therefore, it is difficult to compare our results with other studies since all women had institutional deliveries and all received counselling during the immediate postpartum period. Neonatal care visit was the only independent factor affecting rates of utilization from maternal health services. Our preliminary report showed that the integration of maternal and neonatal services after delivery significantly increased rates of utilization from maternal health care services.

\section{Strengths and limitations of the study}

Most of the data from previous studies were extracted from Demographic Health Surveys of the population or from a retrospective study concerning women who had given birth within five years preceding the investigation $[22,23,24,25,26,27,28]$. Population-based studies have enlightened health policies, however limited number of studies have examined the success of postnatal interventions and their implications on public health. Apart from the other studies, this study investigated the public health implications of integrated maternal and neonatal services. To the best of our knowledge, this paper is the first to examine and share the experiences gained from the integration of PNC with NHT and NC in Turkey. The major strength of the report was the inclusion of all births during the study 
period, thus the paper is far from patient selection bias. However, this paper has some limitations. It is a single center report, therefore the results cannot be generalized. It is also a preliminary-quality report of integrated services rather than a randomized clinical trial. For his reason, further assessments in different settings are needed.

\section{Conclusions}

Decreasing perinatal mortality and addressing inequalities in the distribution of health care services are priorities for all countries. This paper suggested that postnatal education of mothers immediately after birth is an essential primary step to increase maternity service use. Moreover, the integration of maternal and infant care after hospital discharge increases uptake of maternal postnatal care. Determining the right integration strategies in different populations requires further evaluation.

Acknowledgments: The authors declare that any financial support was not accepted for this study, and also gratefully acknowledge the contribution of midwives Nural Seslikaya and Seval Ciftci to data collection process.

Conflict of Interest: No conflict of interest was declared by the authors.

Financial Disclosure: The authors declared that this study has received no financial support.

\section{REFERENCES}

1. WHO Technical Consultation on Postpartum and Postnatal Care. Geneva: WHO Document Production Services; 2010.

2. WHO: Trends in maternal mortality: 1990 to 2010. WHO, UNICEF, UNFPA and World Bank Estimates. Geneva. WHO Document Production Services; 2012.

3. Sibley LM, Sipe TA. Transition to skilled birth attendance: is there a future role for trained traditional birth attendants? J Health Popul Nutr 2006;24:472-8.

4. Lawn JE, Kerber K, Enweronu-Laryea C, Massee Bateman O. Newborn survival in low resource settings--are we delivering? BJOG 2009;116 Suppl 1:49-59.

5. Langlois EV, Miszkurka M, Ziegler D, Karp I, Zunzunegui MV. Protocol for a systematic review on inequalities in postnatal care services utilization in low- and middle-income countries. Syst Rev 2013;2:55.

6. Blenning CE, Paladine $\mathrm{H}$. An approach to the postpartum office visit. Am Fam Physician 2005;72:2491-6.

7. Türkyılmaz AS, Abbasoğlu Özgören A, Yıldız D. Differentials in receiving postpartum care of infants and its determinants in Turkey. Turk J Pediatr 2013;55:172-9.

8. MacArthur C, Winter HR, Bick DE, Knowles H, Lilford R, Henderson $\mathrm{C}$, et al. Effects of redesigned community postnatal care on womens' health 4 months after birth: a cluster randomised controlled trial. Lancet 2002;359:378-85.

9. Demirel G, Tezel B, Ozbas S, Oguz SS, Erdeve O, Uras N, et al. Rapid decrease of neonatal mortality in Turkey. Matern Child Health J 2013;17:1215-21.

10. Turkey Demographic and Health Survey Report 2008. Hacettepe Universtity Institute of Population studies, Ministry of Health Genaral Directorate of Mother and Child Health and Family Planning, T.R. Prime Ministry Undersecretary of State Planning Organisation and TÜBİTAK, Ankara Turkey; 2009.

11. Kültürsay N. The status of women and of maternal and perinatal health in Turkey. Turk J Pediatr 2011;53:5-10.

12. Turkey Demographic and Health Survey Report. 2013. Hacettepe Universtity Institute of Population studies, Ministry of Health Genaral Directorate of Mother and Child Health and Family Planning, T.R. Prime Ministry Undersecretary of State Planning Organisation and TÜBİTAK, Ankara Turkey; 2014.

13. Jafarey S, Kamal I, Qureshi AF, Fikree F. Safe motherhood in Pakistan. Int J Gynaecol Obstet 2008;102:179-85.

14. Ahmed S, Creanga AA, Gillespie DG, Tsui AO. Economic status, education and empowerment: implications for maternal health service utilization in developing countries. PLoS One 2010;5:e11190.

15. Amin R, Shah NM, Becker S. Socioeconomic factors differentiating maternal and child health-seeking behavior in rural Bangladesh: A cross-sectional analysis. Int J Equity Health 2010;9:9.

16. Bhutta ZA, Chopra M, Axelson H, Berman P, Boerma T, Bryce J, et al. Countdown to 2015 decade report (2000-10): taking stock of maternal, newborn, and child survival. Lancet 2010;375:2032-44.

17. Hussein J, Newlands D, D’Ambruoso L, Thaver I, Talukder R, Besana G. Identifying practices and ideas to improve the implementation of maternal mortality reduction programmes: findings from five South Asian countries. BJOG 2010;117:304-13.

18. Ronsmans C, Chowdhury ME, Koblinsky M, Ahmed A. Care seeking at time of childbirth, and maternal and perinatal mortality in Matlab, Bangladesh. Bull World Health Organ 2010;88:289-96.

19. Titaley CR, Dibley MJ, Roberts CL. Factors associated with non-utilisation of postnatal care services in Indonesia. J Epidemiol Community Health 2009;63:827-31.

20. Rakhecha A. Universal neonatal hearing screening: neonatologist's perspective. Indian Pediatr 2014;51:173-4.

21. Bolat H, Bebitoglu FG, Ozbas S, Altunsu AT, Kose MR. National newborn hearing screening program in Turkey: struggles and implementations between 2004 and 2008. Int J Pediatr Otorhinolaryngol 2009;73:1621-3. 
22. Chakraborty N, Islam MA, Chowdhury RI, Bari W. Utilisation of postnatal care in Bangladesh: evidence from a longitudinal study. Health Soc Care Community 2002;10:492-502.

23. Syed U, Asiruddin Sk, Helal MS, Mannan II, Murray J. Immediate and early postnatal care for mothers and newborns in rural Bangladesh. J Health Popul Nutr 2006;24:508-18.

24. Dhakal S, Chapman GN, Simkhada PP, van Teijlingen ER, Stephens J, Raja AE. Utilisation of postnatal care among rural women in Nepal. BMC Pregnancy Childbirth 2007;7:19.

25. Neupane S, Doku D. Utilization of postnatal care among Nepalese women. Matern Child Health J 2013;17:1922-30.
26. Workineh GY, Hailu DA. Factors affecting utilization of postnatal care service in Jabitena district, Amhara region, Ethiopia. Science Journal of Public Health 2014;2:169-76.

27. Khanal V, Adhikari M, Karkee R, Gavidia T. Factors associated with the utilisation of postnatal care services among the mothers of Nepal: analysis of Nepal demographic and health survey 2011. BMC Womens Health 2014;14:19.

28. Yamashita T, Suplido SA, Ladines-Llave C, Tanaka Y, Senba $\mathrm{N}$, Matsuo H. A cross-sectional analytic study of postpartum health care service utilization in the Philippines. PLoS One 2014;9:e85627. 